\title{
Ueber den Uebergang der Bernsteinsäure in den Harn ;
}

von Dr. Wilhelm Hallwachs.

In einer Abhandlung "Ueber den Uebergang organischer Säuren in den Harn" von Buch heim und Piotrowsky*) findet sich die überraschende Angabe, dafs die Bernsteinsäure in den Organismus gebracht nicht, wie es wohl zu erwarten stand, unverändert denselben passire, sondern dafs diese, den chemischen Agentien so kräflig wiederstehende Säure, nachdem sie eingenommen war, weder in dem Harn noch in den festen Excrementen wieder aufgefunden werden konnte. Ich glauble dieses so merkwürdige Resullat durch Versuche controliren zu müssen, zumal da eine frühere Angabe von Wöhler**) vorliegt, nach welcher der unveränderte Uebergang der Bernsteinsäure in den Harn beobachtet wurde ****).

Während ich mit diesen Versuchen beschäfligt war, erschien eine Untersuchung über denselhen Gegenstand von

*) Archiv für physiologische Heilkunde von Wunderlich, 1857. Neue Folge. 1. Band, 1. Heft, S. 42.

**) Diesen Versuch machte ich vor 35 Jahren, mit noch sehr geringer Erfahrung, sehr dürftigen Mitteln und nur ein Nal. Es ist also wahrscheinlich, dafs ich mich geirrt habe. Indessen wäre zu bemerken, dafs der damals untersuchte Hundeharn abnorm beschaffen, nämlich trübe und alkalisch war und mit Säuren stark brauste, während der von Hrn. H. untersuchte Harn schwach sauer reagirte. Mit jener abnormen Beschaffenheit kōnnte aber ein wirklicher Uebergang der Bernsteinsäure im Zusammenhange gestanden haben.

W

*a*) Tiedemann und Treviranus, Zeitschrift f. Plyssiologie, Bd. I, Heft 1, S. 141 . 
Kühn $\mathrm{e}^{*}$ ), welche zu einem noch merkwürdigeren Resultate, wie das von Buch he im war, geführt hatte. Kühne halte nämlich beobachtet, dafs Bernsteinsäure eingenommen eine bedeutende Mehrausscheidung von Hippursäure bewirke.

Selbst bei den kühnsten, fast bis an das Unerlaubte grenzenden Speculationen war es unmöglich, eine Ahnung von dem inneren Wesen dieser eigenthümlichen, ächten Metamorphose zu bekommen. Auch das Resultat von Kühne mufste defshalb einer controlirenden Untersuchung unterworfen werden. Bei Bestätigung desselben war gewifs die Hoffnung nicht unberechtigt, die Erklärung der Hippursäurebildung beim Menschen wie bei den Pflanzenfressern einen bedeutenden Schrilt vorwärts zu bringen, zumal da das Vorkommen der Bernsteinsäure in Organismus zu verschiedenen Malen auf das Bestimmteste erwiesen ist. Heintz*\%), Boedeker ***;) und v. Gorup-Besanezt) haben dieselbe nachgewiesen, die Ersteren in dem wässerigen Inhalt von Echinococcenbälgen, G or up-Bes a nez fand sie normal in der Thymusdrüse des Kalbes, der Thyreoïdea und der Milz des Rindes. Material zur Bildung der Bernsteinsäure ist dem Organismus reichlich geboten in den Fellen, dem Stärkemehl, Zucker und äpfelsauren Salzen der Nahrung.

In wie weit wir es hoffen dürfen, von der Bernsteinsäure Aufklärung über die Hippursäurefrage zu erhalten ist in dem Folgenden dargethan.

Buchheim und Pietrowsky haben, wie ich ihre Mittheilung verstehe, niemals das bemerkte Auftreten der Hippursäure von der eingenommenen Bernsteinsäure abgeleitet,

*) Virchow's Archiv für path. Anat. u. Physiol. 1857, XII, 396-401.

**) Pogg. Ann. LXXX, 114.

***) Henle u. Pfenfer's Zeitschr. f. rationelle Medicin, N. F. VII, 127.

t) Diese Annalen XCVIII, 23. 
wie $\mathrm{K}$ ühn e glaubt, sondern sie haben nur einmal, als der Harnrückstand sorgfältiger, wie bei den anderen Versuchen, mit Aether ausgezogen wurde, stall Bernsteinsäure etwas Hippursäure erhalten.

Meine Versuche, zu deren Beschreibung ich nun übergehe, wurden an einem Hunde angestellt. Diese Thiere als Fleischfresser scheiden in ihrem Harne keine Hippursäure aus, und es durften defshalb sehr scharfe Resultale bei ihnen erwartet werden. Ehe ich mit dem Eingeben der Bernsteinsäure begann, prïfte ich die Güle der Methode, welche ich bei der Harnuntersuchung anzuwenden gedachte. Es hatte diese Methode den Zweck, Hippursäure und Bernsteinsäure aus dem Harn neben einander rein zu erhalten, um alsdann die Kalksalze der beiden Säuren miltelst Alkohol zu trennen, da in diesem wohl der hippursaure, aber nicht der bernsteinsaure Kalk löslich ist.

Einer Menge von $500 \mathrm{CC}$. Hundeharn wurden 2 Gramm bernsteinsaures Natron zugesetzt, der Harn zur Trockene gebracht, mit Schwerspathpulver fein zerrieben, darauf mit Salzsäure angesäuert und mit Alkohol bis zur völligen Erschöpfung ausgezogen. Nach der Neutralisation des so dargestellten alkoholischen Auszuges mit Natronlauge wurde der gröfste Theil des Alkohols abdestillirt, und die zurückbleibende syrupöse Flüssigkeit nach Zusatz von Oxalsäure im Wasserbade, unter Umrühren, zur Trockene gebracht. Die trockene Masse wurde nun erschöpfend mit Aether ausgezogen und die ätherische Lösung fast bis zur Trockene abdestillirt. Der bleibende krystallinische Rückstand wurde zur Entfernung der gelösten Oxalsäure mit Kalkmilch in der Wärme behandelt, abfiltrirt, das Filtrat bis auf ein geringes Volumen eingedunstet und alsdann mit Salzsäure sauer gemacht. Nach einigen Stunden waren die Wände wie der Boden des Schälchens mit einer zusammenhängenden krystal- 
linischen Kruste bedeckt, welche sich als Bernsteinsäure erwies. Die durch Umkrystallisiren gereinigten Krystalle schmolzen beim Erhitzen und sublimirten theilweise. Eisenchlorid brachle in der neutralisirten Lösung einen voluminösen hellrothbraunen, schwefelsaures Kupferoxyd einen hellblauen, im Ueberschufs nicht löslichen Niederschlag hervor. Die Lösung der Krystalle, mit einer Mischung von Weingeist, Ammoniak und Chlorbaryum versetzt, gab einen weilsen Niederschlag.

Die Brauchbarkeit der Methode war somit dargethan, da sich dieselbe, wie bereits bekannt, zum Nachweis der Hippursäure ebenfalls sehr gut eignet. Im Falle des Vorkommens beider Säuren neben einander mufsten dieselben am Ende der Operation zusammen erhalten werden.

Ich gab dem Hunde an verschiedenen Tagen, indem ich mit dem Eingeben von 2 Gramm (zu einem Versuch) begann, und bis zu 15 Gramm stieg, 64 Grm. Bernsteinsäure, welche mit Brod in Breiform gebracht von dem Thiere freiwillig gefressen wurde. Der Harn wurde nun während der nächsten 30 Stunden aufgefangen; er hatte stets eine schwach saure Reaction. Zu 9 verschiedenen Malen habe ich denselben nach der angegebenen Weise untersucht, aber niemals gelang es mir, weder Hippursäure noch Bernsteinsäure darin nachzuweisen. Nach diesen negativen Resultaten, welche die Angaben von Buchheim bestäligen, schien es mir nothwendig, auch die nach einer Bernsteinsäurefütterung entleerten Fäces zu untersuchen, um die verschwundene Bernsteinsäure vielleicht darin wieder aufzufinden.

Nachdem der Hund nochmals 10 Gramm Bernsteinsäure mit Brod bekommen hatte, wurden die Fäces desselben in den folgenden 36 Stunden gesammelt und der Untersuchung unterworfen. Dieselben waren von brauner Farbe und schmieriger Consistenz. Sie wurden mit verdünnter Schwefelsäure 
zerrieben, in einen Kolben gebracht und wiederholt mit Alkohol ausgezogen. Der Alkoholauszug wurde, nachdem er neutralisirt war, zur Syrupsconsistenz gebracht, wieder angesäuert und dann mit Aether erschöpft. Die verdunsteten Auszüge liefsen keine Spur von Bernsteinsäure zurück, Cholestearin war der am meisten bemerkbare Bestandtheil derselben.

Ich stellte nun einen Versuch an mir selbst an. Bei einer vorzugsweisen (nicht ausschliefslichen) Fleischdiät untersuchte ich den in 24 Stunden gelassenen Harn in der $A b-$ sicht, die in demselben enthaltene Menge von Hippursäure annähernd zu bestimmen. Die gesammle $1300 \mathrm{CC}$. betragende Harnmenge wurde eingedampft und dann in derselben Weise behandelt, wie oben beim Nachweis der Bernsteinsäure angegeben ist. Alle Auszüge wurden auf das Sorgfälligste bereitet, der mit Oxalsäure versetzte Rückstand mit grofsen Mengen von Aether drei Tage lang behandelt. Ich erhielt schliefslich beinahe ein Gramm Hippursäure (unrein gewogen).

Ich nahm jetzl am Morgen 4 Gramm Bernsteinsäure in Pillenform, sammelte wieder während der nächsten 24 Stunden meinen Harn und untersuchte iln alsdann. Der Hippursäuregehalt desselben hatte sich nicht bemerkenswerth verändert. Ein zweiter Versuch, bei welchem ich 6 Gramm Bernsteinsäure genommen hatte, gab mir dasselbe Resultat.

Die grofse Menge von Hippursäure, welche ich in meinem Harn gefunden hattle, veranlafste mich, auch denselben von einigen meiner Bekannten zu untersuchen. Ich erhielt stets ganz ähnliche Quantitäten von Hippursäure. Man sieht hieraus, dals der Hippursäuregehalt des normalen Harnes weit bedeutender ist, als man bisher angenommen hat. Weitere Untersuchungen werden diese Angabe gewifs bestätigen. Durch diese scheint mir auch Kühn e's oben erwähntes Resullat völlig erklärbar zu sein. Die Auszüge wurden bei 
der Untersuchung des nach Bernsteinsäuregenuls gelassenen Harnes wahrscheinlich sorgfältiger gemacht, als beim normalen Harn, und so die normale Menge der Hippursäure gefunden, welche $\mathrm{K} \ddot{\mathrm{u} h \mathrm{~h} e}$ nach den bisherigen Angaben allerdings für geringer halten mufste. Wo die eingenommene Bernsteinsäure bleibt, ist nicht erklärt; ob sie zuerst zum Aufbau dem Organismus eigenthümlicher Verbindungen dient, oder vielleicht sofort völlig zu Kohlensäure und Wasser verbrennt, wage ich nicht zu entscheiden.

Götting en, 21. Februar 1858.

\section{Ueber die Aequivalentgewichte des Baryums, des Strontiums und des Blei's; \\ nach C. Marignac *).}

M arignac hat die Aequivalentgewichte des Baryums, des Strontiums und des Blei's durch neue Versuche bestimmt. Baryum. - Das Aequivalentgewicht des Baryums war schon durch mehrere Chemiker, auch durch Marignac selbst, in der Art bestimmt worden, dals gesucht wurde, wie viel Silber zur Ausfällung des Chlors aus einer bekannten Menge wasserfreien Chlorbaryums erforderlich ist. Marignac hat neue Versuche angestellt, welche einerseits diese fruheren Bestimmungen in derselben Weise controliren, andererseits dem möglicherweise zu erhebenden $Z_{w}$ eifel nicht ausgeselzt

*) Im Auszug aus den Archives des sciences physiques et naturelles, Mars 1858. 